\title{
Research Article Synchronization and Pinning Control in Complex Networks with Interval Time-Varying Delay
}

\author{
Hai-Feng Jiang' and Tao $\mathrm{Li}^{\mathbf{2}}$ \\ ${ }^{1}$ School of Automation, Nanjing University of Science and Technology, Nanjing 210094, China \\ ${ }^{2}$ School of Automation Engineering, Nanjing University of Aeronautics and Astronautics, \\ Nanjing 210016, China \\ Correspondence should be addressed to Hai-Feng Jiang, blowingwinds@yahoo.cn
}

Received 24 December 2011; Accepted 16 January 2012

Academic Editor: Teh-Lu Liao

Copyright @ 2012 H.-F. Jiang and T. Li. This is an open access article distributed under the Creative Commons Attribution License, which permits unrestricted use, distribution, and reproduction in any medium, provided the original work is properly cited.

The problems on synchronization and pinning control for complex dynamical networks with interval time-varying delay are investigated and two less conservative criteria are established based on reciprocal convex technique. Pinning control strategies are designed to make the complex networks synchronized. Moreover, the problem of designing controllers can be converted into solving a series of NMIs (nonlinear matrix inequalities) and LMIs (linear matrix inequalities), which reduces the computation complexity when comparing with those present results. Finally, numerical simulations can verify the effectiveness of the derived methods.

\section{Introduction}

During the past decades, complex dynamical networks have increasingly become a focal research topic and received much attention in various fields such as physics, chemistry, and computer science [1-4]. Such systems in the real world usually consist of a large number of highly interconnected dynamical units. Transportation networks, coupled engineering systems, artificial neural networks in human brains, and the Internet are only the typically realistic examples. In order to capture their evolving properties, several famous models have been proposed, such as scale-free networks, random networks, and small world networks.

Recently, the research on synchronization and dynamical behaviors of complex networks has become an important direction, and it was mainly based on the techniques including analyzing topological structure and studying the origin of the intrinsic selforganized dynamics; see [5-7] and references therein. Authors in [5-7] have introduced one uniform dynamic network model and studied the phenomena of synchrony of all network 
states, with some derived conditions that can guarantee the asymptotical synchronization or projective cluster synchronization. Meanwhile, since there inevitably exists communication delay which is the main source of poor dynamical behaviors in various networks, some efforts have been applied to the synchronization of complex systems [8-21]. More recently, some researchers have considered the global or robust or cluster synchronization for various coupled delayed neural networks based on some effective techniques including LMI one in [8-12]. As for complex networks with linear or nonlinear couplings, some delay-dependent criteria are presented to guarantee the synchronization in [13-16], and moreover in $[17,18]$, some synchronization results are achieved for complex networks based on linear feedback control or adaptive feedback control. Additionally, to avoid the conservatism, some authors employed delay-partitioning idea to tackle the constant delay or time-variant one in $[19,20]$. Yet, it has come to our attention that though some delay-dependent methods have appeared, the techniques above still require great improvements owing to ignoring some important terms when estimating the derivative of Lyapunov functional, especially for the case of interval time-varying delay [21].

Presently, the control problem on scale-free dynamical networks was investigated by applying local linear feedback injections to a small fraction of network nodes [22, 23]. Meanwhile, some researchers have considered pinning control of delayed complex networks in [24-31]. In [24], cluster synchronization for stochastic delayed neural networks was studied based on pinning control and LMI approach. By adopting pinning periodically intermittent control, the work [25] studied the exponential synchronization and yet gave some uneasyto-check results. With existence of various couplings, the synchronization has been analyzed for delayed complex networks using pinning adaptive control in [26]. In [27], the authors have presented linear feedback and adaptive feedback pinning control to synchronize the delayed complex networks. Yet, we notice that as for constructions of Lyapunov functional and analyzed techniques described in [24-29], there still exists much room waiting for the further improvement, which can be fully considered and tackled in this presented work.

In this paper, the problems on synchronization and pinning control for delayed dynamical networks are studied based on pinning control strategies. Then the decentralized feedback controllers are designed to make the addressed networks synchronized. Moreover, since the most improved techniques including reciprocal convex technique have been applied, the design of controllers can be converted into solving optimal solution of an array of NMIs and LMIs. These conditions can be extended to delayed complex networks with different topology and different sizes.

Notations. $\mathbf{R}^{n}$ denotes the $n$-dimensional Euclidean space, and $\mathbf{R}^{n \times m}$ is the set of all $n \times m$ real matrices. $A^{T}$ stands for the transpose of matrix $A ; I$ represents the identity matrix of an appropriate dimension; $A \otimes B$ indicates the Kronecker product of the $m \times n$ matrix $A$ and $p \times q$ matrix $B ;\left[\begin{array}{cc}X & Y \\ Y^{T} & Z\end{array}\right]=\left[\begin{array}{ll}X & Y \\ * & Z\end{array}\right]$ with $*$ denoting the symmetric term in a symmetric matrix.

\section{Problem Formulations}

Suppose that the nodes are coupled with states $x_{i}(\cdot), i \in\{1, \ldots, N\}$, then the complex dynamical networks can be described by

$$
\dot{x}_{i}(t)=f\left(x_{i}(t)\right)+g\left(x_{i}(t-\tau(t))\right)+\sum_{j=1}^{N} l_{i j}^{1} E x_{j}(t)+\sum_{j=1}^{N} l_{i j}^{2} F x_{j}(t-\tau(t)),
$$


where $x_{i}(t)=\left[x_{i 1}(t), x_{i 2}(t), \ldots, x_{i n}(t)\right]^{T}$ are the state vectors, and $f(\cdot), g(\cdot): \mathbf{R}^{n} \rightarrow \mathbf{R}^{n}$ are the continuously differentiable functions; here, $E=\left[e_{i j}\right]_{n \times n}$ and $F=\left[f_{i j}\right]_{n \times n}$ are the inner coupling matrices between the connected nodes $i$ and $j$ at time $t$ and $t-\tau(t)$, respectively.

For the dynamical system (2.1), the following assumptions are utilized throughout this paper:

(A1) $\tau(t)$ denotes the interval time-varying delay satisfying

$$
0 \leq \tau_{0} \leq \tau(t) \leq \tau_{m}, \quad \dot{\tau}(t) \leq \mu<+\infty,
$$

and we set $\bar{\tau}_{m}=\tau_{m}-\tau_{0}$,

(A2) here, $L^{k}=\left[l_{i j}^{k}\right]_{N \times N}(k=1,2)$ is the configuration matrix that is irreducible and satisfies

$$
l_{i i}^{k}=-\sum_{j=1, j \neq i}^{N} l_{i j}^{k}=-\sum_{j=1, j \neq i}^{N} l_{j i}^{k}, k=1,2 ; i=1,2, \ldots, N,
$$

with $l_{i j}^{k}=l_{j i}^{k}>0$ if there is a connection between node $i$ and the one $j$, and otherwise, $l_{i j}^{k}=$ 0 . Furthermore, suppose that the eigenvalue $\lambda_{i}\left(v_{i}\right)$ of the matrix $L^{1}\left(L^{2}\right)$ can be ordered as follows:

$$
0=\lambda_{1}>\lambda_{2} \geq \cdots \geq \lambda_{N} \quad\left(0=v_{1}>v_{2} \geq \cdots \geq v_{N}\right)
$$

Prior to addressing the synchronization results, the following definition and lemmas are introduced.

Definition 2.1 (see [28]). The complex networks described by (2.1) are said to achieve the asymptotical synchronization

$$
x_{1}(t)=x_{2}(t)=\cdots=x_{N}(t)=s(t),
$$

where $s(t)$ is the solution of the local dynamics of an isolate node satisfying

$$
\dot{s}(t)=f(s(t))+g(s(t-\tau(t))) .
$$

Lemma 2.2 (see [32]). For any constant matrix $W \in \mathbf{R}^{n \times n}, W=W^{T}>0$, scalar functional $0 \leq$ $r(t) \leq r_{M}$, and a vector function $\dot{e}:\left[-r_{M}, 0\right] \rightarrow \mathbf{R}^{n}$ such that the following integration is well defined, then

$$
-r_{M} \int_{-r(t)}^{0} \dot{e}^{T}(t+s) W \dot{e}(t+s) d s \leq[e(t)-e(t-r(t))]^{T} W[e(t)-e(t-r(t))] .
$$


Lemma 2.3 (see [33]). Let the functions $f_{1}(t), f_{2}(t), \ldots, f_{N}(t): \mathbf{R}^{m} \rightarrow \mathbf{R}$ have the positive values in an open subset $\mathbf{D}$ of $\mathbf{R}^{m}$, then for $\alpha_{i}>0$ satisfying $\sum_{i} \alpha_{i}=1$ and $g_{i, j}(t): \mathbf{R}^{m} \rightarrow \mathbf{R}$ satisfying $\left[\begin{array}{cc}f_{i}(t) & g_{i, j}(t) \\ g_{j, i}(t) & f_{j}(t)\end{array}\right] \geq 0$, one has that

$$
\sum_{i} \frac{1}{\alpha_{i}} f_{i}(t) \geq \sum_{i} f_{i}(t)+\sum_{i \neq j} g_{i, j}(t)
$$

As for the complex networks (2.1) and symmetric matrices $L_{1}, L_{2}$, if the following $N-1$ systems of $n$-dimensional linear delayed differential systems

$$
\dot{\omega}_{i}(t)=\left(J(t)+\lambda_{i} E\right) \omega_{i}(t)+\left(\Gamma(t)+v_{i} F\right) \omega_{i}(t-\tau(t)), \quad i=2, \ldots, N
$$

are asymptotically stable with respect to their zero solutions, the complex networks (2.1) can achieve the synchronization at $s(t)$, where $J(t) \dot{B} f^{\prime}(s(t))$ is the Jacobian matrix of $f(x(t))$ at $s(t)$, and $\Gamma(t) \dot{\mathrm{B}} g^{\prime}(x(t-\tau(t)))$ is the Jacobian matrix of $g(x(t-\tau(t)))$ at $s(t-\tau(t))$.

\section{Synchronization Criterion for Complex Networks}

In this section, we will derive one delay-derivative-dependent synchronization criterion for the complex networks in (2.1), which can be much more effective than those present ones based on some most developed techniques in [28-33].

Theorem 3.1. Considering the time delay in (2.2), the complex networks (2.1) can achieve the asymptotical synchronization if there exist the appropriately dimensional matrices $P_{i}>0, Q_{i}>0$, $R_{i}>0, T_{i}>0, U_{i}>0, V_{i}, S_{i}, H_{i}, M_{i}$, and $N_{i}$ for $i=2, \ldots, N$, satisfying the matrix inequalities in (3.1),

$$
\begin{gathered}
{\left[\begin{array}{cc}
V_{i} & S_{i} \\
S_{i}^{T} & V_{i}
\end{array}\right] \geq 0, \quad i=2, \ldots, N,} \\
{\left[\begin{array}{ccccc}
\Omega_{i 11} & U_{i} & \Omega_{i 13} & 0 & \Omega_{i 15} \\
* & \Omega_{i 22} & V_{i}-S_{i} & S_{i} & 0 \\
* & * & \Omega_{i 33} & V_{i}-S_{i} & \Omega_{i 35} \\
* & * & * & -R_{i}-V_{i} & 0 \\
* & * & * & * & \Omega_{i 55}
\end{array}\right]<0,}
\end{gathered}
$$

with

$$
\begin{aligned}
& \Omega_{i 11}=-U_{i}+Q_{i}+H_{i}^{T}\left(J(t)+\lambda_{i} E\right)+\left(J(t)+\lambda_{i} E\right)^{T} H_{i}, \\
& \Omega_{i 13}=H_{i}^{T}\left(\Gamma(t)+\nu_{i} F\right)+\left(J(t)+\lambda_{i} E\right)^{T} N_{i}, \\
& \Omega_{i 15}=P_{i}-H_{i}^{T}+\left(J(t)+\lambda_{i} E\right)^{T} M_{i}, \\
& \Omega_{i 22}=-Q_{i}+R_{i}+T_{i}-V_{i}-U_{i},
\end{aligned}
$$


Mathematical Problems in Engineering

$$
\begin{aligned}
& \Omega_{i 33}=-(1-\mu) T_{i}-N_{i}^{T}\left(\Gamma(t)+v_{i} F\right)+\left(\Gamma(t)+v_{i} F\right)^{T} N_{i}-2 V_{i}+S_{i}+S_{i}^{T}, \\
& \Omega_{i 35}=\left(\Gamma(t)+v_{i} F\right)^{T} M_{i}-N_{i}^{T} \\
& \Omega_{i 55}=-M_{i}^{T}-M_{i}+\tau_{0}^{2} U_{i}+\bar{\tau}_{m}^{2} V_{i} .
\end{aligned}
$$

Proof. As for the $i$ th node, we choose the following Lyapunov-Krasovskii functional:

$$
\begin{aligned}
V_{i}\left(\omega_{i_{t}}\right)= & \omega_{i}^{T}(t) P_{i} \omega_{i}(t)+\int_{t-\tau_{0}}^{t} \omega_{i}^{T}(s) Q_{i} \omega_{i}(s) d s+\int_{t-\tau_{m}}^{t-\tau_{0}} \omega_{i}^{T}(s) R_{i} \omega_{i}(s) d s+\int_{t-\tau(t)}^{t-\tau_{0}} \omega_{i}^{T}(s) T_{i} \omega_{i}(s) d s \\
& +\int_{-\tau_{0}}^{0} \int_{t+\theta}^{t} \tau_{0} \dot{\omega}_{i}^{T}(s) U_{i} \dot{\omega}_{i}(s) d s d \theta+\int_{-\tau_{m}}^{-\tau_{0}} \int_{t+\theta}^{t} \bar{\tau}_{m} \dot{\omega}_{i}^{T}(s) V_{i} \dot{\omega}_{\mathrm{i}}(s) d s d \theta,
\end{aligned}
$$

with symmetric matrices $P_{i}, Q_{i}, R_{i}, T_{i}, U_{i}$, and $V_{i}$ waiting to be determined. Then for any $n \times n$ matrices $H_{i}, M_{i}$, and $N_{i}$, the time derivative of $V_{i}\left(\omega_{i}(t)\right)$ along the system (2.9) can be derived as

$$
\begin{aligned}
\dot{V}_{i}\left(\omega_{i_{t}}\right)= & 2 \omega_{i}^{T}(t) P_{i} \dot{\omega}_{i}(t)+\omega_{i}^{T}(t) Q_{i} \omega_{i}(t)-\omega_{i}^{T}\left(t-\tau_{0}\right)\left(Q_{i}-R_{i}-T_{i}\right) \omega_{i}\left(t-\tau_{0}\right)-\omega_{i}^{T}\left(t-\tau_{m}\right) R_{i} \omega_{i}\left(t-\tau_{m}\right) \\
& -(1-\dot{\tau}(t)) \omega_{i}^{T}(t-\tau(t)) T_{i} \omega_{i}(t-\tau(t))+\tau_{0}^{2} \dot{\omega}_{i}^{T}(t) U_{i} \dot{\omega}_{i}(t)-\int_{t-\tau_{0}}^{t} \tau_{0} \dot{\omega}_{i}^{T}(s) U_{i} \dot{\omega}_{i}(s) d s \\
& +\bar{\tau}_{m}^{2} \dot{\omega}_{i}^{T}(t) V_{i} \dot{\omega}_{i}(t)-\int_{t-\tau(t)}^{t-\tau_{0}} \bar{\tau}_{m} \dot{\omega}_{i}^{T}(s) V_{i} \dot{\omega}_{i}(s) d s-\int_{t-\tau_{m}}^{t-\tau(t)} \bar{\tau}_{m} \dot{\omega}_{i}^{T}(s) V_{i} \dot{\omega}_{i}(s) d s \\
& +\left[\omega_{i}^{T}(t) H_{i}^{T}+\dot{\omega}_{i}^{T}(t) M_{i}^{T}+\omega_{i}^{T}(t-\tau(t)) N_{i}^{T}\right] \\
& \times\left[-\dot{\omega}_{i}(t)+\left(J(t)+\lambda_{i} E\right) \omega_{i}(t)+\left(\Gamma(t)+v_{i} F\right) \omega_{i}(t-\tau(t))\right] .
\end{aligned}
$$

Through employing Lemmas 2.2 and 2.3, we can yield that

$$
\begin{gathered}
-\int_{t-\tau_{0}}^{t} \tau_{0} \dot{\omega}_{i}^{T}(s) U_{i} \dot{\omega}_{i}(s) d s \leq\left[\omega_{i}(t)-\omega_{i}\left(t-\tau_{0}\right)\right]^{T} U_{i}\left[\omega_{i}(t)-\omega_{i}\left(t-\tau_{0}\right)\right], \\
-\int_{t-\tau(t)}^{t-\tau_{0}} \bar{\tau}_{m} \dot{\omega}_{i}^{T}(s) V_{i} \dot{\omega}_{i}(s) d s-\int_{t-\tau_{m}}^{t-\tau(t)} \bar{\tau}_{m} \dot{\omega}_{i}^{T}(s) V_{i} \dot{\omega}_{i}(s) d s \\
\leq-\frac{\bar{\tau}_{m}}{\tau(t)-\tau_{0}}\left[\omega_{i}\left(t-\tau_{0}\right)-\omega_{i}(t-\tau(t))\right]^{T} V_{i}\left[\omega_{i}\left(t-\tau_{0}\right)-\omega_{i}(t-\tau(t))\right] \\
-\frac{\bar{\tau}_{m}}{\tau_{m}-\tau(t)}\left[\omega_{i}(t-\tau(t))-\omega_{i}\left(t-\tau_{m}\right)\right]^{T} V_{i}\left[\omega_{i}(t-\tau(t))-\omega_{i}\left(t-\tau_{m}\right)\right] \\
\leq-\left[\begin{array}{l}
\omega_{i}\left(t-\tau_{0}\right)-\omega_{i}(t-\tau(t)) \\
\omega_{i}(t-\tau(t))-\omega_{i}\left(t-\tau_{m}\right)
\end{array}\right]^{T}\left[\begin{array}{ll}
V_{i} & S_{i} \\
S_{i}^{T} & V_{i}
\end{array}\right]\left[\begin{array}{l}
\omega_{i}\left(t-\tau_{0}\right)-\omega_{i}(t-\tau(t)) \\
\omega_{i}(t-\tau(t))-\omega_{i}\left(t-\tau_{m}\right)
\end{array}\right] .
\end{gathered}
$$


Then together with the terms (3.4)-(3.5), it can be deduced that

$$
\dot{V}_{i}\left(\omega_{i_{t}}\right) \leq\left[\begin{array}{c}
\omega_{i}(t) \\
\omega_{i}\left(t-\tau_{0}\right) \\
\omega_{i}(t-\tau(t)) \\
\omega_{i}\left(t-\tau_{m}\right) \\
\dot{\omega}_{i}(t)
\end{array}\right]^{T}\left[\begin{array}{ccccc}
\Omega_{i 11} & U_{i} & \Omega_{i 13} & 0 & \Omega_{i 15} \\
* & \Omega_{i 22} & V_{i}-S_{i} & S_{i} & 0 \\
* & * & \Omega_{i 33} & V_{i}-S_{i} & \Omega_{i 35} \\
* & * & * & -R_{i}-V_{i} & 0 \\
* & * & * & * & \Omega_{i 55}
\end{array}\right]\left[\begin{array}{c}
\omega_{i}(t) \\
\omega_{i}\left(t-\tau_{0}\right) \\
\omega_{i}(t-\tau(t)) \\
\omega_{i}\left(t-\tau_{m}\right) \\
\dot{\omega}_{i}(t)
\end{array}\right]
$$

Then there must exist one scalar $\varepsilon>0$ such that $\dot{V}_{i}\left(\omega_{i_{t}}\right) \leq-\varepsilon\left\|\mathrm{P} \omega_{i}(t) \mathrm{P}\right\|<0$ for any $\omega_{i}(t) \neq 0$. Based on the Lyapunov-Krasovskii stability theory, all nodes in complex networks (2.1) are asymptotically synchronized, and it completes the proof.

Remark 3.2. Presently, the convex combination technique has been widely employed to tackle time-varying delay owing to that it could reduce the conservatism more effectively than the previous methods; see [21, 32]. In [33], the authors put forward the reciprocal convex approach, which can reduce the conservatism more effectively than convex combination ones. Yet, it has come to our attention that no researchers have utilized reciprocal convex idea to tackle the synchronization for delayed complex networks.

\section{Pinning Control of Delayed Complex Networks}

Suppose that we want to stabilize the system (2.1) onto a homogeneous stationary state. In order to achieve the goal, we will apply the pinning control strategy on a small fraction $\delta(0<\delta \ll 1)$ of the nodes in network (2.1). Suppose that the nodes $i_{1}, i_{2}, \ldots, i_{l}$ are selected to be under pinning control, and $i_{l+1}, i_{l+2}, \ldots, i_{N}$ are the unselected ones, where $l=[\delta N]$ stands for the smaller but nearest to the real number $\delta N$, then $l$ is no less than 0 for pinning control, and the controlled networks can be described by

$$
\begin{aligned}
& \dot{x}_{i}(t)=f\left(x_{i}(t)\right)+g\left(x_{i}(t-\tau(t))\right)+\sum_{j=1}^{N} l_{i j}^{1} E x_{j}(t)+\sum_{j=1}^{N} l_{i j}^{2} F x_{j}(t-\tau(t))+u_{i}(t), \quad i=1,2, \ldots, l, \\
& \dot{x}_{i}(t)=f\left(x_{i}(t)\right)+g\left(x_{i}(t-\tau(t))\right)+\sum_{j=1}^{N} l_{i j}^{1} E x_{j}(t)+\sum_{j=1}^{N} l_{i j}^{2} F x_{j}(t-\tau(t)), \quad i=l+1, l+2, \ldots, N .
\end{aligned}
$$

For a special case that $\tau(t)$ is available, we adopt the following local linear feedback control law:

$$
u_{i}(t)=-k_{i} E\left[x_{i}(t)-s(t)\right]-l_{i} F\left[x_{i}(t-\tau(t))-s(t-\tau(t))\right]
$$

with the feedback gains $k_{i}>0, l_{i}>0$.

The aim of this section is to stabilize the network (4.1) to be of homogenous stationary state defined by

$$
x_{1}(t), \quad x_{2}(t), \ldots, x_{N}(t) \longrightarrow s(t), \quad \dot{s}(t)=f(s(t))+g(s(t-\tau(t))), \quad \text { as } t \longrightarrow+\infty .
$$


Denoting $k_{i}=l_{i}=0(i=l+1, l+2, \ldots, N)$, then we can derive that

$$
\begin{aligned}
\dot{x}_{i}(t)= & f\left(x_{i}(t)\right)+g\left(x_{i}(t-\tau(t))\right)+\sum_{j=1}^{N} l_{i j}^{1} E x_{j}(t)+\sum_{j=1}^{N} l_{i j}^{2} F x_{j}(t-\tau(t))-k_{i} E\left[x_{i}(t)-s(t)\right] \\
& -l_{i} F\left[x_{i}(t-\tau(t))-s(t-\tau(t))\right], \quad i=1,2, \ldots, N .
\end{aligned}
$$

If we let $e_{i}(t)=x_{i}(t)-s(t)$ and $e(t)=\left[e_{1}(t), e_{2}(t), \ldots, e_{N}(t)\right]^{T}$, then we can linearize the network (4.4) as

$\dot{e}_{i}(t)=J(t) e_{i}(t)+\Gamma(t) e_{i}(t-\tau(t))+\sum_{j=1}^{N} l_{i j}^{1} E e_{j}(t)+\sum_{j=1}^{N} l_{i j}^{2} F e_{j}(t-\tau(t))-k_{i} E e_{i}(t)-l_{i} F e_{i}(t-\tau(t))$,

where $J(t)$ and $\Gamma(t)$ are the Jacobian matrices of the functions $f, g$ at $s(t)$ and $s(t-\tau(t))$, respectively.

In what follows, we define $K=\operatorname{diag}\left(k_{1}, k_{2}, \ldots, k_{N}\right), L=\operatorname{diag}\left(l_{1}, l_{2}, \ldots, l_{N}\right)$, and

$$
L^{1}=\left[l_{i j}^{1}\right]_{N \times N^{\prime}} \quad L^{2}=\left[l_{i j}^{2}\right]_{N \times N^{\prime}} \quad e(t)=\left[e_{1}^{T}(t), e_{2}^{T}(t), \ldots, e_{N}^{T}(t)\right]^{T}
$$

Then based on Kronecker product, we can deduce

$$
\dot{e}(t)=\left(I_{N} \otimes J(t)\right) e(t)+\left(I_{N} \otimes \Gamma(t)\right) e(t-\tau(t))+\left(\left(L^{1}-K\right) \otimes E\right) e(t)+\left(\left(L^{2}-L\right) \otimes F\right) e(t-\tau(t)) .
$$

Assumption 4.1. The matrices $L^{1}-K$ and $L^{2}-L$ satisfy $\left[\left(L^{1}-K\right)\left(L^{2}-L\right)\right]^{T}=\left(L^{1}-K\right)\left(L^{2}-L\right)$.

Then there does exist an orthogonal matrix $U$ such that $U\left(L^{1}-K\right) U^{T}=\bar{\Lambda}$ and $U\left(L^{2}-\right.$ $L) U^{T}=\bar{\Pi}$, in which $\bar{\Lambda}=\operatorname{diag}\left(\bar{\lambda}_{1}, \bar{\lambda}_{2}, \ldots, \bar{\lambda}_{N}\right)$ and $\bar{\Pi}=\operatorname{diag}\left(\bar{v}_{1}, \bar{v}_{2}, \ldots, \bar{v}_{N}\right)$ satisfy $0>\bar{\lambda}_{1} \geq$ $\bar{\lambda}_{2} \geq \cdots \geq \bar{\lambda}_{N}$ and $0>\bar{v}_{1} \geq \bar{v}_{2} \geq \cdots \geq \bar{v}_{N}$.

Denoting $\eta(t)=\left(U^{T} \otimes I_{n}\right) e(t)=\left[\eta_{1}^{T}(t), \eta_{2}^{T}(t), \ldots, \eta_{N}^{T}(t)\right]^{T}$ and employing the property of Kronecker product $\left(A_{1} \otimes B_{1}\right)\left(A_{2} \otimes B_{2}\right)=\left(A_{1} A_{2}\right) \otimes\left(B_{1} B_{2}\right)$, one can derive

$$
\dot{\eta}(t)=\left(I_{N} \otimes J(t)\right) \eta(t)+\left(I_{N} \otimes \Gamma(t)\right) \eta(t-\tau(t))+(\bar{\Lambda} \otimes E) \eta(t)+(\bar{\Pi} \otimes F) \eta(t-\tau(t)),
$$

that is,

$$
\dot{\eta}_{i}(t)=\left[J(t)+\bar{\lambda}_{i} E\right] \eta_{i}(t)+\left[\Gamma(t)+\bar{v}_{i} F\right] \eta_{i}(t-\tau(t)), \quad i=1,2, \ldots, N
$$

Theorem 4.2. Considering the time delay in (2.2), the controlled networks (4.9) can achieve local asymptotical stability at $s(t)$ if there exist the appropriately dimensional matrices $P_{i}>0, Q_{i}>0$, 
$R_{i}>0, T_{i}>0, U_{i}>0, V_{i}>0, S_{i}, H_{i}, M_{i}$, and $N_{i}$ for $i=1,2, \ldots, N$, satisfying the following inequalities in (4.10):

$$
\begin{gathered}
{\left[\begin{array}{cc}
V_{i} & S_{i} \\
S_{i}^{T} & V_{i}
\end{array}\right] \geq 0, \quad i=1,2, \ldots, N,} \\
{\left[\begin{array}{ccccc}
\Xi_{i 11} & U_{i} & \Xi_{i 13} & 0 & \Xi_{i 15} \\
* & \Xi_{i 22} & V_{i}-S_{i} & S_{i} & 0 \\
* & * & \Xi_{i 33} & V_{i}-S_{i} & \Xi_{i 35} \\
* & * & * & -R_{i}-V_{i} & 0 \\
* & * & * & * & \Xi_{i 55}
\end{array}\right]<0,}
\end{gathered}
$$

with

$$
\begin{aligned}
& \Xi_{i 11}=-U_{i}+Q_{i}+H_{i}^{T}\left(J(t)+\bar{\lambda}_{i} E\right)+\left(J(t)+\bar{\lambda}_{i} E\right)^{T} H_{i}, \\
& \Xi_{i 13}=H_{i}^{T}\left(\Gamma(t)+\bar{v}_{i} F\right)+\left(J(t)+\bar{\lambda}_{i} E\right)^{T} N_{i}, \\
& \Xi_{i 15}=P_{i}-H_{i}^{T}+\left(J(t)+\bar{\lambda}_{i} E\right)^{T} M_{i}, \\
& \Xi_{i 22}=-Q_{i}+R_{i}+T_{i}-V_{i}-U_{i}, \\
& \Xi_{i 33}=-(1-\mu) T_{i}-N_{i}^{T}\left(\Gamma(t)+\bar{v}_{i} F\right)+\left(\Gamma(t)+\bar{v}_{i} F\right)^{T} N_{i}-2 V_{i}+S_{i}+S_{i}^{T}, \\
& \Xi_{i 35}=\left(\Gamma(t)+\bar{v}_{i} F\right)^{T} M_{i}-N_{i}^{T}, \\
& \Xi_{i 55}=-M_{i}^{T}-M_{i}+\tau_{0}^{2} U_{i}+\bar{\tau}_{m}^{2} V_{i},
\end{aligned}
$$

where $\bar{\lambda}_{i}$ and $\bar{v}_{i}$ are the ith eigenvalues of the matrices $L^{1}-K$ and $L^{2}-L$, respectively.

Proof. As for the $i$ th node, we choose the following Lyapunov-Krasovskii functional:

$$
\begin{aligned}
V_{i}\left(\eta_{i_{t}}\right)= & \eta_{i}^{T}(t) P_{i} \eta_{i}(t)+\int_{t-\tau_{0}}^{t} \eta_{i}^{T}(s) Q_{i} \eta_{i}(s) d s+\int_{t-\tau_{m}}^{t-\tau_{0}} \eta_{i}^{T}(s) R_{i} \eta_{i}(s) d s+\int_{t-\tau(t)}^{t-\tau_{0}} \eta_{i}^{T}(s) T_{i} \eta_{i}(s) d s \\
& +\int_{-\tau_{0}}^{0} \int_{t+\theta}^{t} \tau_{0} \dot{\eta}_{i}^{T}(s) U_{i} \dot{\eta}_{i}(s) d s d \theta+\int_{-\tau_{m}}^{-\tau_{0}} \int_{t+\theta}^{t} \bar{\tau}_{m} \dot{\eta}_{i}^{T}(s) V_{i} \dot{\eta}_{i}(s) d s d \theta
\end{aligned}
$$

with appropriately dimensional matrices $P_{i}>0, Q_{i}>0, R_{i}>0, T_{i}>0, U_{i}>0, V_{i}>0$ waiting to be determined.

Together with similar proof of Theorem 3.1, one can yield

$$
\dot{V}_{i}\left(\eta_{i_{t}}\right) \leq\left[\begin{array}{c}
\eta_{i}(t) \\
\eta_{i}\left(t-\tau_{0}\right) \\
\eta_{i}(t-\tau(t)) \\
\eta_{i}\left(t-\tau_{m}\right) \\
\dot{\eta}_{i}(t)
\end{array}\right]^{T}\left[\begin{array}{ccccc}
\Xi_{i 11} & U_{i} & \Xi_{i 13} & 0 & \Xi_{i 15} \\
* & \Xi_{i 22} & V_{i}-S_{i} & S_{i} & 0 \\
* & * & \Xi_{i 33} & V_{i}-S_{i} & \Xi_{i 35} \\
* & * & * & -R_{i}-V_{i} & 0 \\
* & * & * & * & \Xi_{i 55}
\end{array}\right]\left[\begin{array}{c}
\eta_{i}(t) \\
\eta_{i}\left(t-\tau_{0}\right) \\
\eta_{i}(t-\tau(t)) \\
\eta_{i}\left(t-\tau_{m}\right) \\
\dot{\eta}_{i}(t)
\end{array}\right] .
$$


Then there does exist one scalar $\varepsilon>0$ such that $\dot{V}_{i}\left(\eta_{i}(t)\right) \leq-\varepsilon\left\|\mathrm{P} \eta_{i}(t) \mathrm{P}\right\|<0$ for any $\eta_{i}(t) \neq 0$. Based on the theory of Lyapunov stability, the controlled network (4.9) is locally stable for any time-delay satisfying (2.2). The proof is completed.

Remark 4.3. Based on Theorem 4.2, for the controlled dynamical network with fixed network structure and coupling strength as in (4.1), it is easy to determine the number of pinned nodes for network synchronization and select nodes to pin since there are no restrictions for the positions of the pinned nodes. Moreover, the reciprocal convex technique can reduce the conservatism more effectively than those earlier methods.

\section{Numerical Examples}

Now, two numerical examples with simulations will be presented to illustrate efficiency of our results.

Example 5.1. Consider one 3-node complex network, in which each node is a simple 3dimensional nonlinear system [28],

$$
\left[\begin{array}{l}
\dot{x}_{i 1}(t) \\
\dot{x}_{i 2}(t) \\
\dot{x}_{i 3}(t)
\end{array}\right]=\left[\begin{array}{c}
-x_{i 1}(t)+x_{i 2}^{2}(t) \\
-2 x_{i 2}(t) \\
-3 x_{i 3}(t)+x_{i 2}(t) x_{i 3}(t)
\end{array}\right], \quad i=1,2,3
$$

which is asymptotically stable at $s(t)=0$, and its Jacobian matrix is $J=\operatorname{diag}(-1,-2,-3)$. Assuming that the inner coupling matrices and the coupling configuration ones can be represented as

$$
E=F=\left[\begin{array}{lll}
1 & 0 & 0 \\
0 & 1 & 0 \\
0 & 0 & 1
\end{array}\right], \quad L^{1}=L^{2}=\left[\begin{array}{ccc}
-1 & 0.5 & 0.5 \\
0.5 & -1 & 0.5 \\
0.5 & 0.5 & -1
\end{array}\right]
$$

then the eigenvalues of matrices $L^{1}, L^{2}$ can be computed as $0,-1.5,-1.5$, respectively.

In the case of $\tau_{0}=\mu=0$, using [28, Theorem 1] in gives $\tau_{\max }=0.615$, while applying theorem in this paper can verify that the maximum delay upper bound guaranteeing the synchronized state to be asymptotically stable is $\tau_{\max }=0.867$. This means that our results can be less conservative than some existing ones.

When $\tau(t)=\sin ^{2}(2 t)$, it follows from Theorem 3.1 that the feasible solution to the condition in (3.1) can be existent. Defining the total error of the array of the system (5.1) as follows:

$$
\operatorname{error}(t)=\sum_{i=1}^{3} \sqrt{\left[x_{1 i}(t)-x_{2 i}(t)\right]^{2}+\left[x_{2 i}(t)-x_{3 i}(t)\right]^{2}},
$$

then the synchronization error can be seen in Figure 1, and it shows that system (5.1) can achieve the asymptotical synchronization. During the process of simulation, the initial conditions of nodes are selected as $x_{1}=[-0.5,-0.3,0.3]^{T}, x_{2}=[0.7,-0.5,-0.6]^{T}$, and $x_{3}=$ $[1,0.5,0.3]^{T}$. 


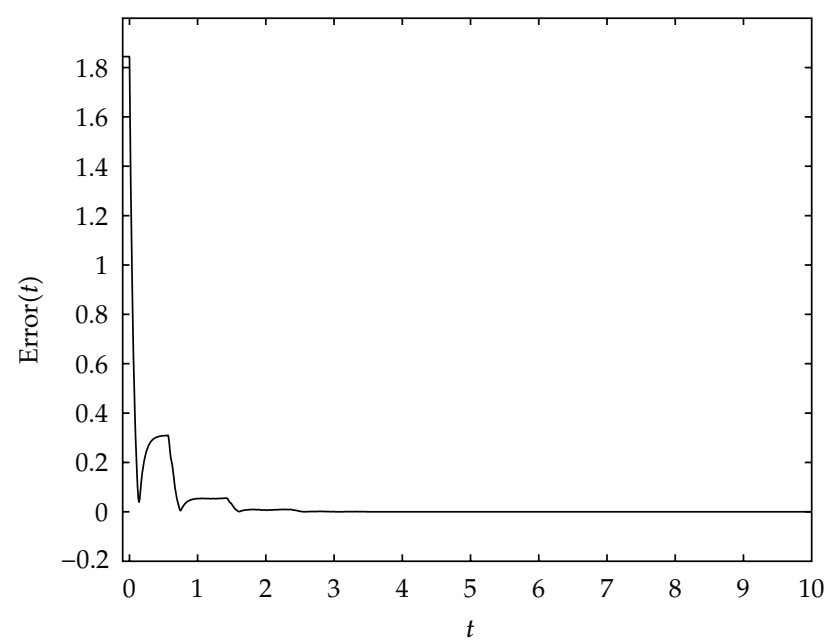

Figure 1: The total synchronous error of system (5.1).

Example 5.2. Together with local linear feedback pinning control in (4.2) to the first node and setting feedback gains $k_{1}=l_{1}=1$, we consider the following nonlinear system,

$$
\left[\begin{array}{c}
\dot{x}_{i 1}(t) \\
\dot{x}_{i 2}(t)
\end{array}\right]=\left[\begin{array}{c}
-x_{i 1}(t)+x_{i 2}^{2}(t) \\
-3 x_{i 1}(t)+x_{i 1}(t) x_{i 2}(t)
\end{array}\right]+\left[\begin{array}{l}
2 x_{i 1}(t-\tau(t))-x_{i 2}(t-\tau(t)) \\
x_{i 1}(t-\tau(t))+3 x_{i 2}(t-\tau(t))
\end{array}\right], \quad i=1,2,3
$$

with $\tau(\mathrm{t})=\cos ^{2}(2 t)$. Its Jacobians are $J=\operatorname{diag}(-1,-3)$ and $\Gamma=\left[\begin{array}{cc}2 & -1 \\ 1 & 3\end{array}\right]$, respectively. The inner coupling matrices and the coupling configuration ones can be represented as

$$
E=F=\left[\begin{array}{ll}
1 & 0 \\
0 & 1
\end{array}\right], \quad L^{1}=L^{2}=\left[\begin{array}{ccc}
-1 & 0.5 & 0.5 \\
0.5 & -1 & 0.5 \\
0.5 & 0.5 & -1
\end{array}\right]
$$

Then we can stabilize the network onto the original equilibrium point $\bar{s}=[0,0,0]^{T}$ based on Theorem 4.2, and the feasible solution to (4.10) can be existent with the eigenvalues of matrices $L^{1}-K$ and $L^{2}-L$ being $\{-2.9808,-1.5000,-0.2192\}$. We also define the total error of the array of system (5.4) as follows:

$$
\operatorname{error}(t)=\sum_{i=1}^{2} \sqrt{\left[x_{1 i}(t)-x_{2 i}(t)\right]^{2}+\left[x_{2 i}(t)-x_{3 i}(t)\right]^{2}}
$$

then the synchronization error can be seen in Figure 2, and it shows that system (5.4) can achieve the asymptotical synchronization. During the process of simulation, the initial conditions of nodes are selected as $x_{1}=[-0.5,-0.3]^{T}, x_{2}=[0.7,-0.5]^{T}$, and $x_{3}=[0.4,0.3]^{T}$. 


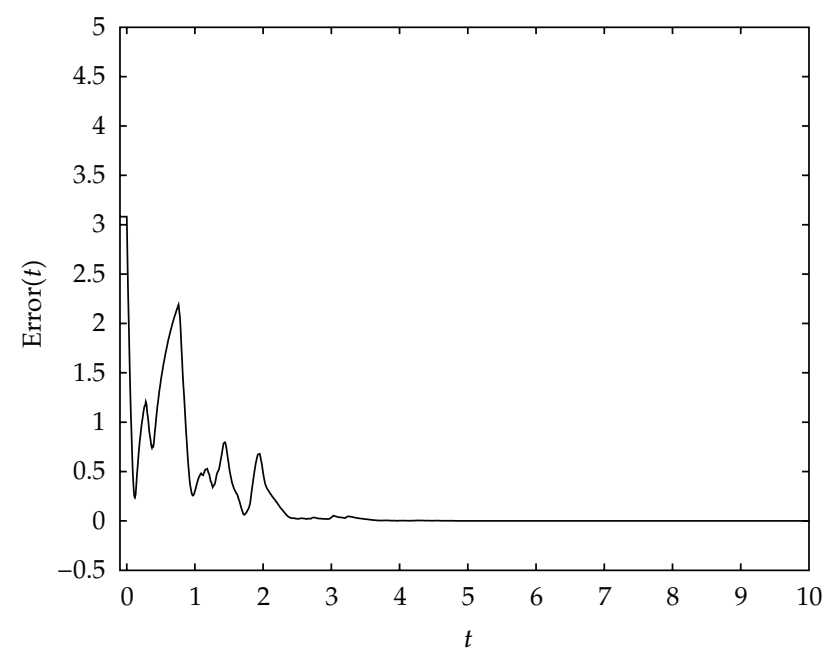

Figure 2: The total synchronous error of system (5.4).

\section{Conclusions}

In this paper, the asymptotical synchronization and pinning control for delayed dynamical networks have been studied based on Lyapunov-Krasovskii functional theory, and the decentralized feedback controller has been designed to make the networks synchronized. Moreover, since the most improved techniques including reciprocal convex one have been applied, the established results can be more applicable than those present ones, and the design of controllers can be achieved by solving an array of NMIs and LMIs. Finally, two illustrative examples can show the efficiency of the proposed methods.

\section{Acknowledgment}

This work is supported by National Natural Science Foundation of China under Grant 61004032 .

\section{References}

[1] A.-L. Barabási and R. Albert, "Emergence of scaling in random networks," American Association for the Advancement of Science, vol. 286, no. 5439, pp. 509-512, 1999.

[2] S. N. Dorogovtesev and J. F. F. Mendes, "Evolution of networks," Advances in Physics, vol. 51, no. 4, pp. 1079-1087, 2002.

[3] M. E. J. Newman, "The structure and function of complex networks," SIAM Review, vol. 45, no. 2, pp. 167-256, 2003.

[4] S. Boccaletti, V. Latora, Y. Moreno, M. Chavez, and D.-U. Hwang, "Complex networks: structure and dynamics," Physics Reports, vol. 424, no. 4-5, pp. 175-308, 2006.

[5] X. F. Wang and G. Chen, "Synchronization in scale-free dynamical networks: robustness and fragility," IEEE Transactions on Circuits and Systems I, vol. 49, no. 1, pp. 54-62, 2002.

[6] X. F. Wang and G. Chen, "Synchronization in small-world dynamical networks," International Journal of Bifurcation and Chaos in Applied Sciences and Engineering, vol. 12, no. 1, pp. 187-192, 2002.

[7] M. F. Hu, Z. Y. Xu, and Y. Q. Yang, "Projective cluster synchronization in drive-response dynamical networks," Physica A, vol. 387, no. 14, pp. 3759-3768, 2008. 
[8] M. F. Hu, Y. Q. Yang, and Z. Y. Xu, "Cluster synchronization in an array of hybrid coupled neural networks with delay," Physica A, vol. 381, no. 15, pp. 457-466, 2007.

[9] Y. Tang and J.-A. Fang, "Robust synchronization in an array of fuzzy delayed cellular neural networks with stochastically hybrid coupling," Neurocomputing, vol. 72, no. 13-15, pp. 3253-3262, 2009.

[10] Q. K. Song, "Synchronization analysis in an array of asymmetric neural networks with time-varying delays and nonlinear coupling," Applied Mathematics and Computation, vol. 216, no. 5, pp. 1605-1613, 2010.

[11] Q. K. Song, "Synchronization analysis of coupled connected neural networks with mixed time delays," Neurocomputing, vol. 72, no. 16-18, pp. 3907-3914, 2009.

[12] M. De la Sen, "Sufficiency-type stability and stabilization criteria for linear time-invariant systems with constant point delays," Acta Applicandae Mathematicae, vol. 83, no. 3, pp. 235-256, 2004.

[13] D. H. Ji, J. H. Park, W. J. Yoo, S. C. Won, and S. M. Lee, "Synchronization criterion for Lur'e type complex dynamical networks with time-varying delay," Physics Letters Section A, vol. 374, no. 10, pp. 1218-1227, 2010.

[14] S. Y. Xu and Y. Yang, "Synchronization for a class of complex dynamical networks with time-delay," Communications in Nonlinear Science and Numerical Simulation, vol. 14, no. 8, pp. 3230-3238, 2009.

[15] X. J. Wu and H. T. Lu, "Exponential synchronization of weighted general delay coupled and nondelay coupled dynamical networks," Computers \& Mathematics with Applications, vol. 60, no. 8, pp. 2476-2487, 2010.

[16] T. Liu, J. Zhao, and D. J. Hill, "Synchronization of complex delayed dynamical networks with nonlinearly coupled nodes," Chaos, Solitons and Fractals, vol. 40, no. 3, pp. 1506-1519, 2009.

[17] H. Pan, X.-H. Nian, and W.-H. Gui, "Synchronization in dynamic networks with time-varying delay coupling based on linear feedback controllers," Acta Automatica Sinica, vol. 36, no. 12, pp. 1766-1772, 2010.

[18] Y. Z. Xiao, W. Xu, and X. C. Li, "Synchronization of chaotic dynamical network with unknown generally time-delayed couplings via a simple adaptive feedback control," Communications in Nonlinear Science and Numerical Simulation, vol. 15, no. 2, pp. 413-420, 2010.

[19] Y. Wang, Z. D. Wang, and J. L. Liang, "A delay fractioning approach to global synchronization of delayed complex networks with stochastic disturbances," Physics Letters Section A, vol. 372, no. 39, pp. 6066-6073, 2008.

[20] X. J. Wu and H. T. Lu, "Exponential synchronization of weighted general delay coupled and nondelay coupled dynamical networks," Computers $\mathcal{E}$ Mathematics with Applications, vol. 60, no. 8, pp. 2476-2487, 2010.

[21] D. Yue and H. J. Li, "Synchronization stability of continuous/discrete complex dynamical networks with interval time-varying delays," Neurocomputing, vol. 73, no. 4-6, pp. 809-819, 2010.

[22] X. F. Wang and G. Chen, "Pinning control of scale-free dynamical networks," Physica A, vol. 310, no. 3-4, pp. 521-531, 2002.

[23] X. Li, X. F. Wang, and G. R. Chen, "Pinning a complex dynamical network to its equilibrium," IEEE Transactions on Circuits and Systems I, vol. 51, no. 10, pp. 2074-2087, 2004.

[24] L. L. Li and J. D. Cao, “Cluster synchronization in an array of coupled stochastic delayed neural networks via pinning control," Neurocomputing, vol. 74, no. 5, pp. 846-856, 2011.

[25] S. M. Cai, J. J. Hao, Q. B. He, and Z. Liu, "Exponential synchronization of complex delayed dynamical networks via pinning periodically intermittent control," Physics Letters Section A, vol. 375, no. 19, pp. 1965-1971, 2011.

[26] Z. Y. Wang, L. H. Huang, Y. N. Wang, and Y. Zuo, "Synchronization analysis of networks with both delayed and non-delayed couplings via adaptive pinning control method," Communications in Nonlinear Science and Numerical Simulation, vol. 15, no. 12, pp. 4202-4208, 2010.

[27] J. C. Zhao, J.-A. Lu, and Q. J. Zhang, "Pinning a complex delayed dynamical network to a homogenous trajectory," IEEE Transactions on Circuits and Systems II, vol. 56, no. 6, pp. 514-518, 2009.

[28] D. G. Xu and Z. F. Su, "Synchronization criterions and pinning control of general complex networks with time delay," Applied Mathematics and Computation, vol. 215, no. 4, pp. 1593-1608, 2009.

[29] Z. X. Liu, Z. Q. Chen, and Z. Z. Yuan, "Pinning control of weighted general complex dynamical networks with time delay," Physica A, vol. 375, no. 1, pp. 345-354, 2007.

[30] S. Zheng, "Adaptive-impulsive control of the projective synchronization in drive-response complex dynamical networks with time-varying coupling," Mathematical Problems in Engineering, vol. 2012, Article ID 501843, 12 pages, 2012. 
[31] H. Zhang, T. Li, and S. Fei, "Synchronization for an array of coupled Cohen-Grossberg neural networks with time-varying delay," Mathematical Problems in Engineering, vol. 2011, Article ID 831695, 22 pages, 2011.

[32] H. Shao, "New delay-dependent stability criteria for systems with interval delay," Automatica, vol. 45, no. 3, pp. 744-749, 2009.

[33] P. Park, J. W. Ko, and C. Jeong, "Reciprocally convex approach to stability of systems with timevarying delays," Automatica, vol. 47, no. 1, pp. 235-238, 2011. 


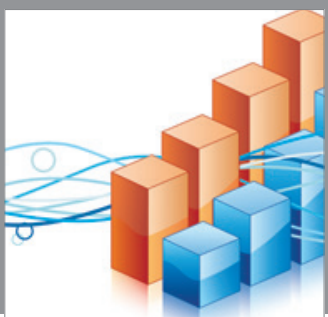

Advances in

Operations Research

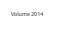

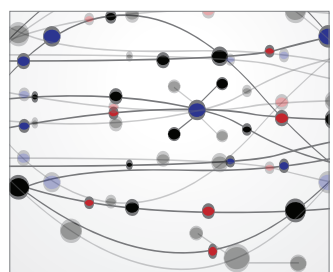

\section{The Scientific} World Journal
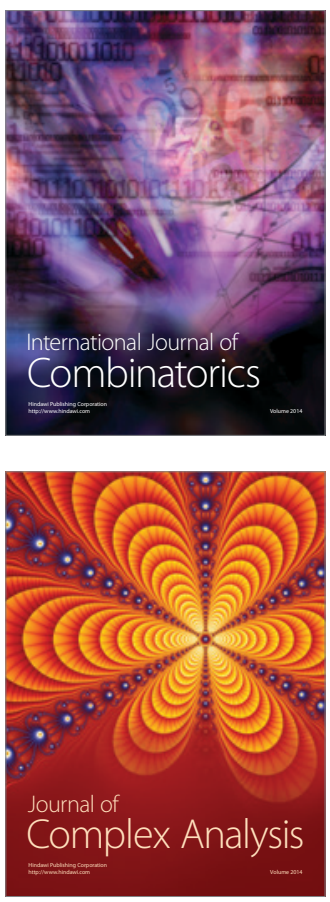

International Journal of

Mathematics and

Mathematical

Sciences
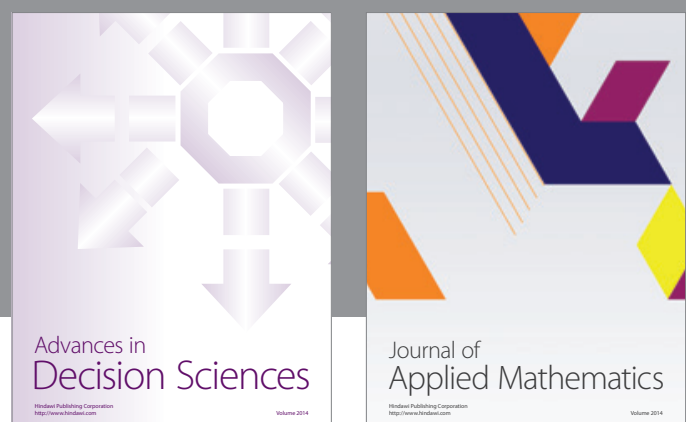

Journal of

Applied Mathematics
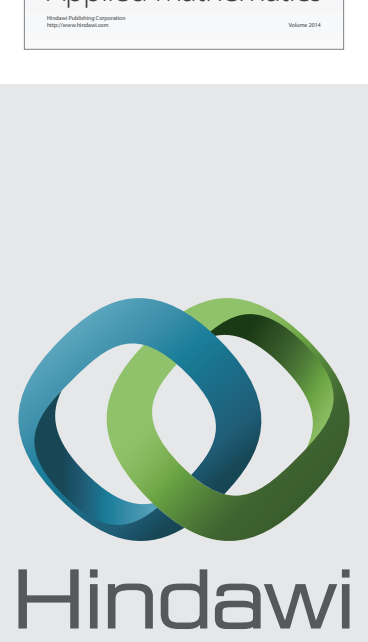

Submit your manuscripts at http://www.hindawi.com
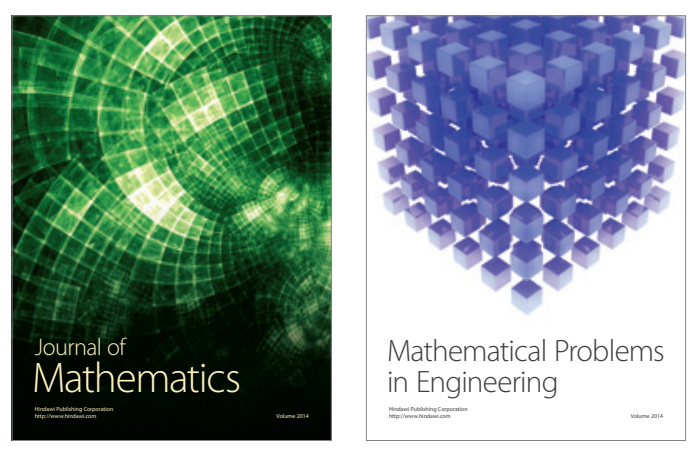

Mathematical Problems in Engineering
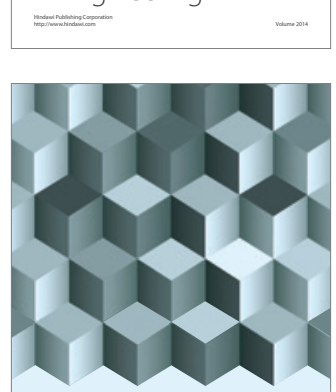

Journal of

Function Spaces
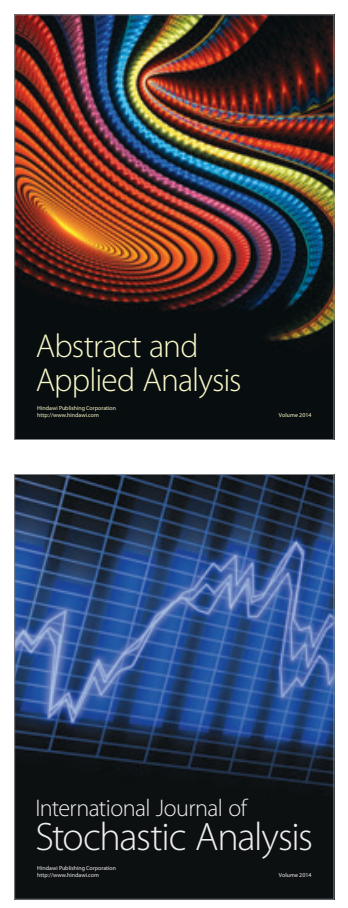

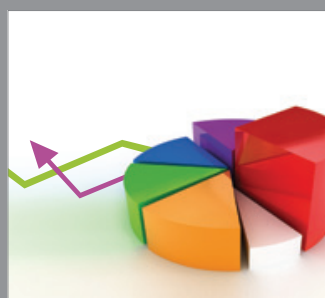

ournal of

Probability and Statistics

Promensencen
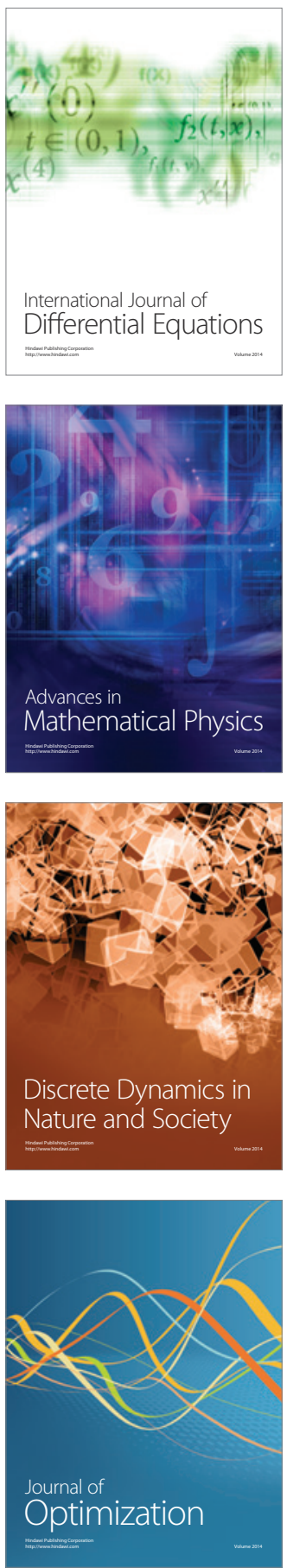